Journal for ImmunoTherapy of Cancer \section{medical record (EMR) to triage patients
admitted with high-grade immune-
related adverse events (irAEs) to the
immune-toxicity (ITOX) service \\ Novel platform leveraging electronic}

To cite: Abu-Shawer 0 , Singh $P$, Yenulevich $\mathrm{E}$, et al. Novel platform leveraging electronic medical record (EMR) to triage patients admitted with highgrade immune-related adverse events (irAEs) to the immunetoxicity (ITOX) service. Journal for ImmunoTherapy of Cancer 2020;8:e000992. doi:10.1136/ jitc-2020-000992

- Additional material is published online only. To view please visit the journal online (http://dx.doi.org/10.1136/jitc2020-000992).

Accepted 05 July 2020

Check for updates

(C) Author(s) (or their employer(s)) 2020. Re-use permitted under CC BY-NC. No commercial re-use. See rights and permissions. Published by BMJ.

${ }^{1}$ Harvard Medical School, Boston, Massachusetts, USA ${ }^{2}$ Oncology, Dana Farber Cancer Institute, Boston, Massachusetts, USA

${ }^{3}$ Internal Medicine, Brigham and Women's Hospital, Boston, Massachusetts, USA

${ }^{4}$ Dermatology, Brigham and Women's Hospital, Boston,

Massachusetts, USA

Correspondence to Dr Osama Rahma; osamae_rahma@dfci.harvard. edu

Osama Abu-Shawer (10 , ${ }^{1}$ Prabhsimranjot Singh, ${ }^{1,2,3}$ Eric Yenulevich, ${ }^{2,3}$

Amanda Brito, ${ }^{1,2,3}$ Jian Ni, ${ }^{3}$ Raja-Elie E Abdulnour, ${ }^{1,2,3}$ Shilpa Grover, ${ }^{1,2,3}$ Michael Manos, ${ }^{2}$ Peter Bowling, ${ }^{2}$ Nicole R LeBoeuf, ${ }^{1,2,4}$ Patrick Ott, ${ }^{1,2,3}$ F Stephen Hodi, ${ }^{1,2,3}$ Joseph Jacobson, ${ }^{1,2,3}$ Osama Rahma ${ }^{1,2,3}$

\section{ABSTRACT}

Background The incidence of high-grade immunerelated adverse events (irAEs) due to immune checkpoint inhibitors (ICls) is increasing due to the rapid expansion of indications for their use. There is an urgent need for a feasible approach of identifying patients with high-grade irAEs to ensure early detection and proper management of this unique set of toxicities.

Methods We established one of the first inpatient services that are specifically devoted to mitigating irAEs. The service uses a multidisciplinary approach with consulting service from experts in managing irAEs. We are leveraging the electronicmedical record (EMR) to triage patients who are admitted to the hospital and have received or are currently receiving ICls. A list of patients with $\mathrm{ICl}$ exposure is generated daily by EMR and then curated manually to identify patients with potential irAEs.

Results A total of 129 patients with high-grade irAEs were admitted between June 2018 and June 2019. The most common irAEs were colitis (32\%), pneumonitis $(30 \%)$, and hepatitis (14\%). Eighty five per cent of the patients had grade 3 irAEs and 15\% had grade 4-5. About half of the patients had received ICI monotherapy; $30 \%$ had received combination of ICls and non-ICls; and $19 \%$ had received a combination of ICls. Only $9 \%$ of patients had steroid-refractory irAEs requiring other immunosuppressive agents. The average length of stay for irAE-related admission was 11 days with a readmission rate due to recurrent irAEs of $26 \%$ within a year. Conclusion We demonstrated the feasibility of using the EMR to accurately triage patients with suspected irAEs to a dedicated immune-toxicity service. Our model is adaptable in major academic centers and could have a major impact on quality of care and future clinical research addressing irAEs.

\section{BACKGROUND}

Immunotherapy has recently taken cancer therapy into a new era due to the expansion of immune checkpoint inhibitors (ICIs) activities and indications. ${ }^{1-3}$ As the field of cancer immunotherapy rapidly evolves, greater understanding of the mechanisms of ICI-induced toxicities is needed. Several mechanisms have been implicated including direct binding of the antibodies to cytotoxic T lymphocyte-associated antigen 4 (CTLA-4) on normal tissue (eg, pituitary gland), antigen cross-reactivity between tumors and healthy tissue, increased humoral response of pre-existing autoantibodies, and the induction of pro-inflammatory cytokines. ${ }^{45}$ The patterns, incidence, and severity of immunerelated adverse events (irAEs) vary between programmed death-ligand 1 (PD-L1), programmed death-1 (PD-1), and CTLA-4 inhibitors with higher incidence of grade 3-4 irAEs using CTLA-4 antibodies as single agents $(30 \%-40 \%)$ or in combination with PD-1 antibodies (50\%) compared with single agent PD-1 or PD-L1 inhibitors $(10 \%-20 \%) .^{6-9}$ However, the real-world data (RWD) revealed even higher incidence of specific irAEs (eg, pneumonitis) compared with what has been reported in clinical trials. This highlights the importance of collecting RWD as patient characteristics differ between the general population and patients on trials. ${ }^{10}$ As the field of immunotherapy moves toward combinatorial regimens, the incidence of irAEs is expected to increase, which is concerning as severe irAEs can lead to treatment interruption, discontinuation and can be fatal. ${ }^{11}$

Realizing the unmet need to identify and appropriately treat patients with irAEs, the Immune Toxicity Work Group at Dana-Farber Cancer Institute (DFCI) and Brigham and Women's Hospital (BWH) 
Table 1 The clinical characteristics of patients

\begin{tabular}{ll}
\hline Age median (range) & $65(25-87)$ \\
Gender & \\
Male & $65(50 \%)$ \\
Female & $64(50 \%)$
\end{tabular}

Tumor type

Thoracic 41 (32\%)

Genitourinary $30(23 \%)$

Skin $24(19 \%)$

Breast $11(9 \%)$

Gastrointestinal $10(8 \%)$

Head and neck $6(5 \%)$

Endocrine $5(3 \%)$

Others

$2(1 \%)$

Treatment modality

Monotherapy $66(51 \%)$

\begin{tabular}{|c|c|}
\hline Pembrolizumab & 33 \\
\hline Nivolumab & 25 \\
\hline Atezolizumab & 4 \\
\hline Durvalumab & 4 \\
\hline Ipilimumab & 1 \\
\hline Combination of ICl and non-ICI & $39(30 \%)$ \\
\hline ICl and chemotherapy & 22 \\
\hline $\begin{array}{l}\text { Pembrolizumab, carboplatin, and pemetrexed } \\
\text { Pembrolizumab and eribulin }\end{array}$ & 15 \\
\hline $\begin{array}{l}\text { Pembrolizumab, fluorouracil, leucovorin, and } \\
\text { oxaliplatin }\end{array}$ & 6 \\
\hline
\end{tabular}

$\mathrm{ICl}$ and targeted therapy $\quad 7$

Pembrolizumab and glembatumumab vedotin 2

Pembrolizumab, abemaciclib, and anastrozole 1

Pembrolizumab and axitinib 1

Pembrolizumab and anti-cancer stem cells ${ }^{*} \quad 1$

Atezolizumab and cabozantinib $\quad 1$

Atezolizumab and bromodomain inhibitor 1

$\mathrm{ICl}$ and immunotherapy 5

Nivolumab and conjugated IL-2* 2

Nivolumab and anti-CSF1 ${ }^{\star} \quad 1$

Nivolumab and cancer vaccine ${ }^{*} \quad 1$

$\mathrm{ICl}$ and anti-angiogenesis 3

Pembrolizumab and anti-angiopoietins ${ }^{*} \quad 1$

Atezolizumab and bevacizumab 1

Nivolumab and bevacizumab 1

$\mathrm{ICl}$ and others 2

Pembrolizumab and radium-223 2

Combination of ICls

$24(19 \%)$

Ipilimumab and nivolumab 21

Ipilimumab and pembrolizumab $\quad 1$

Ipilimumab and PD-L1 inhibitor $\quad 1$

Durvalumab and tremelimumab $\quad 1$

Prior $\mathrm{ICl}$ treatment

No

$112(87 \%)$

Continued
Table 1 Continued

\begin{tabular}{lc} 
Yes & $17(13 \%)$ \\
Ipilimumab and nivolumab & 9 \\
Pembrolizumab & 8 \\
Nivolumab & 1 \\
Radiotherapy exposure & \\
Concurrent radiotherapy & $7(5 \%)$ \\
Recent radiotherapy exposure (within a year) & $22(17 \%)$ \\
No recent radiotherapy exposure & $100(78 \%)$ \\
Treatment discontinuation, causes & $118(90 \%)$ \\
Toxicity & $95(81 \%)$ \\
Progression of disease & $16(14 \%)$ \\
Death & $4(3 \%)$ \\
Unknown & $3(2 \%)$ \\
\hline
\end{tabular}

*Investigational drug.

CSF1R, colony-stimulating factor 1 receptor; ICl, immune checkpoint inhibitor; IL-2, interleukin-2 .

was established. This work group consists of experts in the field of immunotherapy and medicine subspecialists (gastroenterology, pulmonology, endocrinology, dermatology, rheumatology, nephrology, cardiology, and neurology) with expertize in managing toxicities in oncology patients. Subsequently, we developed and implemented clinical management guidelines of irAEs based on the published American Society of Clinical Oncology (ASCO) and National Comprehensive Cancer Network (NCCN) guidelines which were modified as per the recommendations of our toxicity experts. ${ }^{12} 13$ The Immune Toxicity Work Group launched the immunetoxicity (ITOX) service in June 2018, an inpatient service that is devoted to treating patients who are admitted with irAEs. In order to rapidly detect and properly treat patients with high-grade irAEs, we leveraged the electronic medical record (EMR) platform, Epic, to identify patients with potential irAEs and triage them to the ITOX service. Here, we describe our unique experience including the methodology used, the patterns of irAEs observed, the characteristics of toxicity management, treatment course, and outcomes.

\section{Methods}

ITOX service structure

An automated daily list of all patients who have received ICIs, have a cancer diagnosis, and were either admitted to inpatient medical oncology or evaluated in the EMR is generated in Epic. This list is then reviewed each morning by a physician assistant (PA), and patients admitted for potential irAEs are then triaged to the ITOX service (online supplemental figure 1). ITOX service is one out of 13 inpatient oncology services at DFCI/BWH. The ITOX service is staffed by two PAs and a medical oncologist and supported by specialists from all medicine subspecialties with expertize in the management of relevant irAEs. 


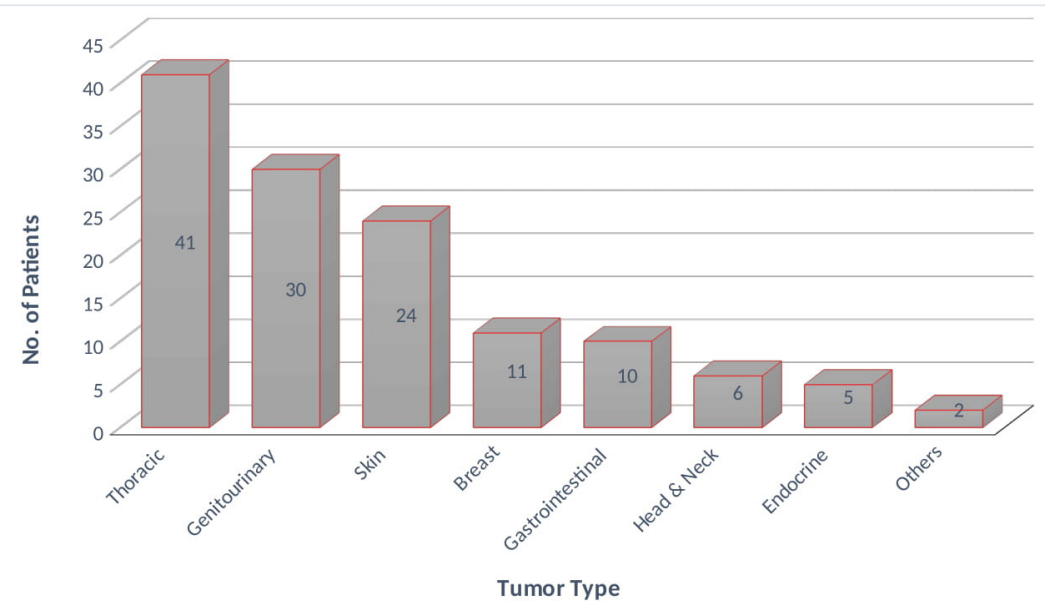

Figure 1 Tumor type. Others include glioblastoma multiforme and sarcoma.

\section{Data collection and analysis}

We performed a retrospective chart review that was approved by the Institutional Review Board (IRB) at DFCI and BWH to report our experience during the first year since the inception of ITOX. We included all cancer patients who were treated with any type of ICI (ipilimumab, tremelimumab, pembrolizumab, nivolumab, atezolizumab, avelumab, or durvalumab) as a part of their standard of care treatment or on clinical trial, as single agents or in combination with other therapies, and were admitted to BWH due to high-grade irAEs between June 2018 and June 2019. Clinical data including patients and tumor characteristics such as primary tumor, current cancer treatment regimens, concurrent or recent radiotherapy exposure, prior cancer treatment were extracted from the charts. The admission data including number and length of irAE-related admissions, and the admitting inpatient services (ITOX, non-ITOX, or intensive care unit (ICU)) were collected. IrAE data included type(s) of irAEs, Common Terminology Criteria for Adverse Events (CTCAE) V.5.0 grade of irAE, imaging and pathology findings (if any), systemic steroids and/or immunosuppressive therapy administration, and cause(s) of cancer treatment discontinuation (due to irAEs or not). If the grades of irAEs were not documented in the chart by the primary team, the data abstractor who is an oncologist or medical specialist graded the irAEs using the NIH CTCAE V.5.0. In the absence of alternative diagnoses, irAEs were confirmed pathologically or clinically by marked response to steroids and/or other immunosuppressive agents. The designation of 'multiple irAEs' was defined as having irAEs involving $\geq 2$ organs at the time of admission. The data were reviewed by a second medical reviewer to ensure its quality and stored in a REDCap database.

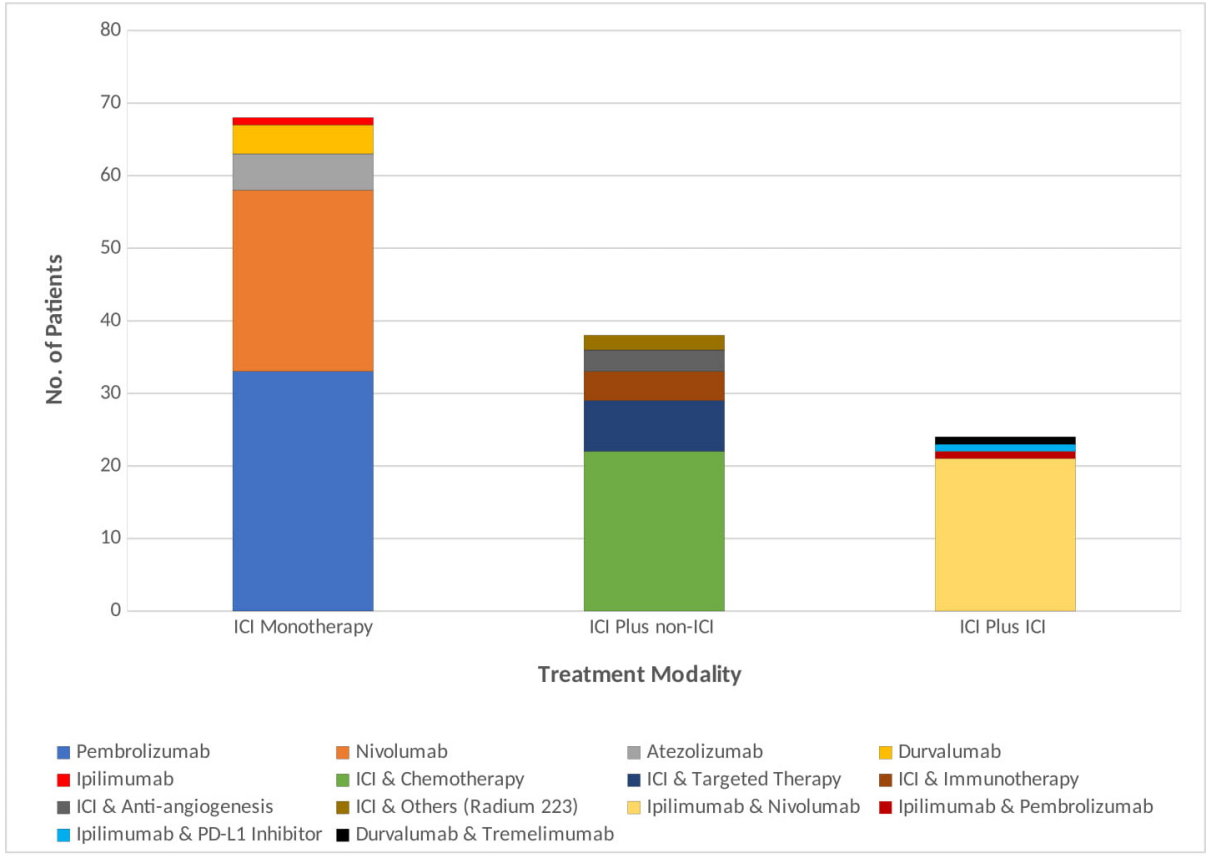

Figure 2 Treatment modality. ICl, immune checkpoint inhibitor. 
Table 2 Immune-related adverse events

$\begin{array}{ll}\text { Number of irAEs } & \\ \text { One irAE } & 97(75 \%) \\ \text { Multiple irAEs } & 32(25 \%) \\ \text { ICI monotherapy } & 11 \\ \text { ICI and non-ICI } & 10 \\ I C I \text { and ICI } & 11\end{array}$

Type of irAEs

\begin{tabular}{|c|c|}
\hline Colitis/enteritis & $55(32 \%)$ \\
\hline Pneumonitis & $51(30)$ \\
\hline Hepatitis & $23(14 \%)$ \\
\hline Thyroiditis & $8(5 \%)$ \\
\hline Myocarditis & $5(3 \%)$ \\
\hline Dermatitis & $5(3 \%)$ \\
\hline Myositis & $4(2 \%)$ \\
\hline Arthritis & $4(2 \%)$ \\
\hline Hypophysitis & $4(2 \%)$ \\
\hline Adrenal insufficiency & $3(2 \%)$ \\
\hline Nephritis & $2(1 \%)$ \\
\hline Pancreatitis & $2(1 \%)$ \\
\hline Flare of underlying autoimmune disease & $2(1 \%)$ \\
\hline Others & $2(1 \%)$ \\
\hline \multicolumn{2}{|l|}{ CTCAE grade of irAEs } \\
\hline Grade 3 (severe) & $110(85 \%)$ \\
\hline Grade 4 (life-threatening) & $14(11 \%)$ \\
\hline Grade 5 (fatal) & $5(4 \%)$ \\
\hline \multicolumn{2}{|l|}{ Hospital course } \\
\hline Length of hospital stay (mean) & 11 days \\
\hline Patients admitted to ICU & $11(8 \%)$ \\
\hline Length of ICU stay (mean) & 7 days \\
\hline \multicolumn{2}{|l|}{ Management of irAEs } \\
\hline Methylprednisolone & $102(80 \%)$ \\
\hline Prednisone & $21(17 \%)$ \\
\hline Dexamethasone & $2(1.5 \%)$ \\
\hline Hydrocortisone & $2(1.5 \%)$ \\
\hline Steroids-refractory irAEs & $12(9 \%)$ \\
\hline Infliximab & 6 \\
\hline IVIG & 2 \\
\hline Infliximab and IVIG & 1 \\
\hline Mycophenolate mofetil & 2 \\
\hline Vedolizumab & 1 \\
\hline \multicolumn{2}{|l|}{ Dose of steroids } \\
\hline$<1 \mathrm{mg} / \mathrm{kg} /$ day & $10(8 \%)$ \\
\hline$=1 \mathrm{mg} / \mathrm{kg} / \mathrm{day}$ & $79(62 \%)$ \\
\hline$>1 \mathrm{mg} / \mathrm{kg} /$ day & $38(30 \%)$ \\
\hline \multicolumn{2}{|l|}{ The irAEs-related admissions } \\
\hline Total number of admissions & 194 \\
\hline
\end{tabular}

Continued
Table 2 Continued

$\begin{array}{ll}\text { ITOX service } & 140(72 \%) \\ \text { Other services } & 54(28 \%) \\ \text { Readmission rate } & 26.00 \%\end{array}$

CTCAE, common Terminology Criteria for Adverse Events; $\mathrm{ICl}$, immune checkpoint inhibitor; ICU, intensive care unit; irAEs, immune-related adverse events; ITOX, immune toxicity; IVIG, intravenous immunoglobulin.

\section{Biospecimen collection}

The ITOX service established an IRB-approved biospecimen banking protocol to collect blood and tissue samples from patients at the time of suspected irAE. Patients eligible for this protocol were identified by reviewing the ITOX inpatient list daily by the clinical coordinators. The patients were then approached during their hospitalization and provided with information on the study; if a patient was willing to participate, the informed consent is obtained, and specimens collected as appropriate.

\section{Results}

\section{Patients characteristics}

A unique 129 patients who received at least one course of ICIs at DFCI were admitted to inpatient medical oncology with high-grade irAEs between June 2018 and June 2019. The clinical characteristics of the patients are summarized in table 1 . Half of the patients were men with a median age of 65 years. The most common tumor types were thoracic $(30 \%)$, genitourinary $(24 \%)$, skin $(18 \%)$, breast (9\%), gastrointestinal (8\%), head \& neck (5\%), endocrine $(3 \%)$, and others (1\%) (figure 1). Half of the patients (51\%) received ICI monotherapy (anti-PD-1 86\%, anti-PD-L1 11\%, anti-CTLA-4 3\%), a one-third (30\%) of the patients received a combination of ICI and non-ICI therapy, and $19 \%$ of the patients received a combination of ICIs (anti-PD-1 and anti-CTLA-4) (figure 2). In 13\% of patients, prior treatment regimens included ICIs (antiPD-1 monotherapy or in combination with anti-CTLA-4). Twenty-three per cent of patients had been exposed to radiotherapy concurrently with ICI $(5 \%)$ or within a year prior to the initiation of ICI $(17 \%)$. Around $90 \%$ of patients discontinued ICI treatment as a result of either toxicity $(81 \%)$, progression of the disease $(14 \%)$, or death $(3 \%)$.

The clinical patterns and management course of irAEs

There was a total of 194 irAE-related admissions between June 2018 and June 2019, 72\% of admissions were appropriately triaged to the ITOX service and the rest to the other inpatient services (table 2). The most common types of irAEs were colitis/enteritis (32\%), pneumonitis (28\%), and hepatitis (13\%) (figure 3). Twenty-five per cent of patients had multiple irAEs at the time of admission (figure 4). Most patients $(85 \%)$ had severe (CTCAE Grade 3) irAEs, while $11 \%$ of the patients had 


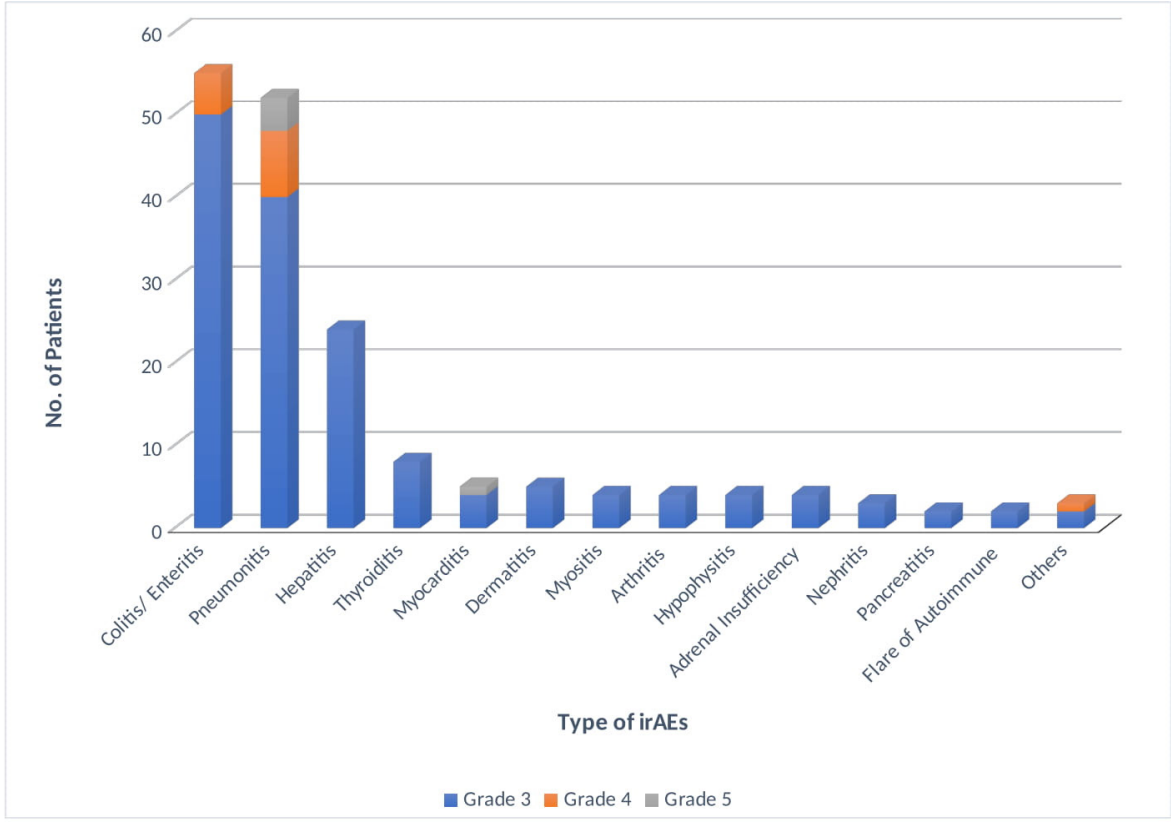

Figure 3 Immune-related adverse events (irAEs). Others include encephalitis, Lambert Eaton syndrome, and polymyalgia rheumatica.

life-threatening (CTCAE Grade 4) irAEs, and only 4\% of the patients developed fatal (CTCAE Grade 5) irAEs (figure 5). Of fatal irAEs, the most common toxicity was pneumonitis which occurred in four out of five cases, and there was one case of myocarditis. Of cases defined as life-threatening, patients presented with pneumonitis (8) colitis (5), and encephalitis (1). Most patients with irAEs received steroid immunosuppression (98\%). Of those receiving steroid immunosuppression, methylprednisolone was given most frequently $(80 \%)$ and steroid dose at initiation was greater than or equal to $1 \mathrm{mg} / \mathrm{kg} /$ day in $92 \%$. Around $9 \%$ of the patients had steroid-refractory irAEs and were treated with non-steroid immunosuppressants (Infliximab, intravenous immunoglobulin (IVIG), mycophenolate mofetil, and vedolizumab). Eight per cent of the patients required admission to the ICU and the average length of hospital stay for all patients was 11 days. The readmission rate due to irAEs within a year was
26\% (table 2). Gastroenterology and pulmonology were the most frequently consulted medical subspecialists (online supplemental figure 2).

\section{Discussion}

For the many cancer patients treated with ICIs, irAEs represent a serious challenge and there is an imminent need to fully understand the patterns of these toxicities and improve their management. Establishing specialized clinical services to ensure early diagnosis and proper management of irAEs can improve the quality of care for patients while also generating data for future research in this field.

We report a 1-year experience of an inpatient service devoted to the care and management of patients with irAEs. We also demonstrate for the first time the feasibility of leveraging the EMR to triage those patients to a specialized toxicity service in a semiautomated system with an

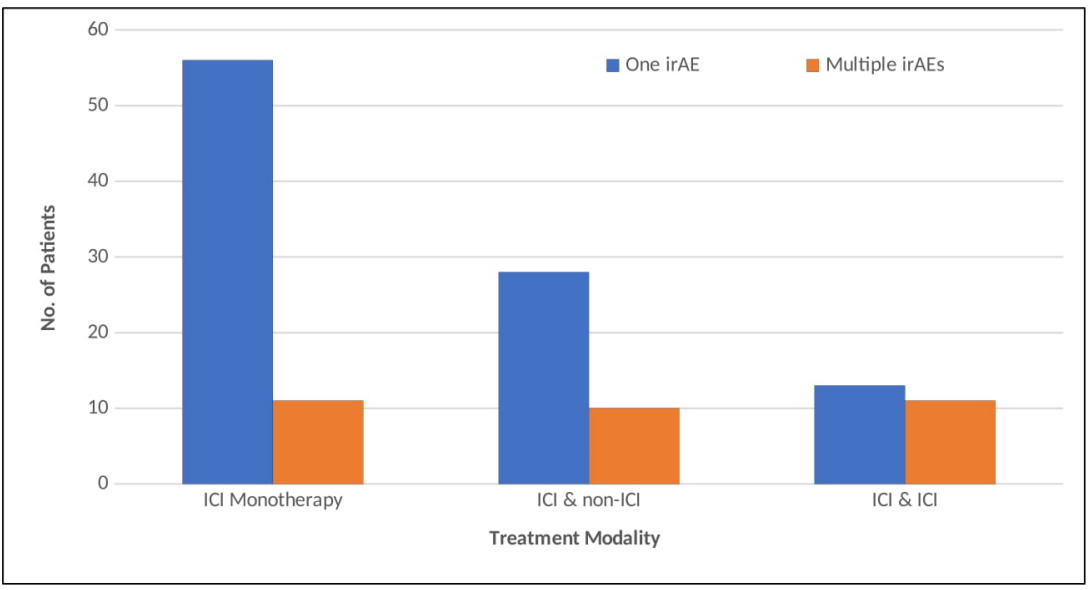

Figure 4 Number of immune-related adverse events (irAEs) based on treatment modality. ICI, immune checkpoint inhibitor. 


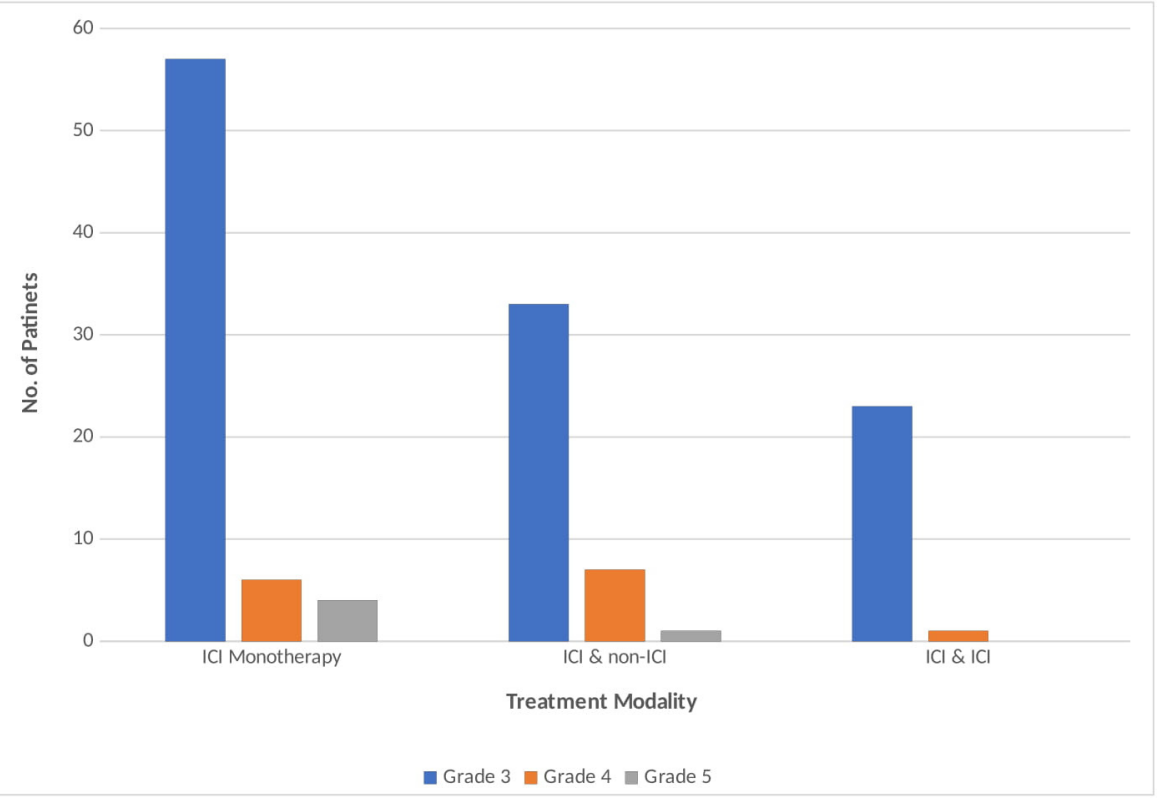

Figure 5 Grade of irAEs based on treatment modality. ICl, immune checkpoint inhibitor; irAEs, immune-related adverse events.

accuracy rate of $71 \%$. The distribution and patterns of the reported irAEs on the ITOX service is reflective of what is expected in the inpatient setting with colitis, hepatitis, and pneumonitis cases accounting for most toxicities and most patients responding to steroids with less than $10 \%$ requiring other immune suppressive agents.

There have been several attempts on the national level to develop programs devoted to treat patients with irAEs. Our partners at Massachusetts General Hospital established the Severe Immunotherapy Complications program which uses the same EMR tool to triage patients with irAEs. The ITOX service at Johns Hopkins University (JHU) published a report about their experience in the Journal of the National Comprehensive Cancer Network. ${ }^{14}$ However, since the ITOX service at JHU is mainly a consulting service on an outpatient basis, their report included mainly patients with low-grade irAEs and fewer patients with high-grade irAEs. ${ }^{15}$

Serious irAEs require prompt immunosuppressive therapy-delays in which may be dangerous. Patients who experience irAEs may seek care from clinicians across specialties, ranging from medical oncology practitioners to primary care, emergency medicine, hospital medicine, and medical subspecialists. Certain irAEs may present with vague symptoms and be particularly challenging to identify for healthcare providers unaccustomed to managing them. The early detection of high-grade irAEs is crucial to facilitate timely initiation of appropriate therapy for these serious adverse events and prevent their long-term complications. Therefore, establishing inpatient services devoted to managing irAEs may improve early detection and proper management. These services will also be helpful in capturing RWD which will advance our knowledge about this increasingly important set of unique toxicities.

There are several limitations to our approach including the remaining need for manual triaging process and the fact that our cohort consists of patients with high-grade irAEs treated at a major academic cancer center where experts in both immunotherapy and immunotoxicity are available, which may not be available in other centers. In addition, establishing such toxicity service requires a lot of resources and funding. Hence, the limitation of adapting this approach elsewhere exists.

Our next steps are to create validated clinical definitions of the most common high-grade irAEs (colitis, pneumonitis, and hepatitis) using the clinical data, diagnostic results, and the patterns of treatment response to improve the accuracy in diagnosing and managing these conditions. We also aim to leverage the wealth of the available data of the patients who developed high-grade irAEs and those who did not experience high-grade irAEs to build a prediction model to identify the patients who are at higher risk of developing high-grade irAEs which will assist oncologists in choosing the most appropriate treatment regimen for these patients when immunotherapy and other modalities are both feasible. The knowledge gained from these projects will be essential to guide the daily practice in both the academic and community settings. In addition, we aim to explore the pathobiology of high-grade irAEs using the available biospecimens obtained at the time of toxicity and before initiating steroids in most cases. This approach may uncover novel therapeutic targets to prevent and treat irAEs.

As cancer immunotherapy continues to evolve with novel single agents and combinations, the burden of irAEs will continue to rise. Therefore, generating easily interpretable clinical definitions of different types of irAEs and building models to detect and predict the occurrence of irAEs are urgently needed to equip healthcare providers with the best strategies to rapidly recognize, diagnose, and manage this increasingly important set of toxicities. Identifying clinical and laboratory-based factors associated with irAEs may also lead to preventive measures to 
decrease the rate of toxicities and guide future immunotherapy drug development. We believe that our model to identify and triage patients with irAEs is a novel platform that, strengthened by continued improvement, could be adaptable at other major academic centers.

\section{Twitter Osama Rahma @0samaRahma2}

Contributors OA-S, PS, AB, SG, R-EEA, NRL, OR contributed to data collection, data analysis, interpretation of the results and writing of the manuscript. MM, $\mathrm{PB}$, $\mathrm{EY}, \mathrm{JN}$ co-write the manuscript and data collection. PO, FSH, JJ, OR contributed to supervision, interpretation of the results and final provision of the manuscript.

Funding The authors have not declared a specific grant for this research from any funding agency in the public, commercial or not-for-profit sectors.

Competing interests None declared.

Patient consent for publication Not required.

Provenance and peer review Not commissioned; externally peer reviewed.

Data availability statement Data are available upon reasonable request. All data relevant to the study are included in the article or uploaded as supplementary information.

Open access This is an open access article distributed in accordance with the Creative Commons Attribution Non Commercial (CC BY-NC 4.0) license, which permits others to distribute, remix, adapt, build upon this work non-commercially, and license their derivative works on different terms, provided the original work is properly cited, appropriate credit is given, any changes made indicated, and the use is non-commercial. See http://creativecommons.org/licenses/by-nc/4.0/

\section{ORCID iD}

Osama Abu-Shawer http://orcid.org/0000-0003-3904-2673

\section{REFERENCES}

1 Gentzler R, Hall R, Kunk PR, et al. Beyond melanoma: inhibiting the PD-1/PD-L1 pathway in solid tumors. Immunotherapy 2016;8:583-600.
2 Postow MA, Callahan MK, Wolchok JD. Immune checkpoint blockade in cancer therapy. J Clin Oncol 2015;33:1974-82.

3 Tang J, Yu JX, Hubbard-Lucey VM, et al. Trial watch: the clinical trial landscape for PD1/PDL1 immune checkpoint inhibitors. Nat Rev Drug Discov 2018;17:854-5.

4 Postow MA, Sidlow R, Hellmann MD, et al. Immune-Related adverse events associated with immune checkpoint blockade. N Engl J Med 2018;378:158-68.

5 Iwama S, De Remigis A, Callahan MK, et al. Pituitary expression of CTLA-4 mediates hypophysitis secondary to administration of CTLA4 blocking antibody. Sci Trans/ Med 2014;6:230ra45.

6 Weber JS, Hodi FS, Wolchok JD, et al. Safety profile of nivolumab monotherapy: a pooled analysis of patients with advanced melanoma. J Clin Oncol 2017;35:785-92.

7 Weber JS, Kähler KC, Hauschild A. Management of immune-related adverse events and kinetics of response with ipilimumab. J Clin Oncol 2012;30:2691-7.

8 Hodi FS, O'Day SJ, McDermott DF, et al. Improved survival with ipilimumab in patients with metastatic melanoma. $N$ Engl $J$ Med 2010;363:711-23.

9 Topalian SL, Hodi FS, Brahmer JR, et al. Safety, activity, and immune correlates of anti-PD-1 antibody in cancer. N Engl $J$ Med 2012;366:2443-54

10 Naidoo J, Wang X, Woo KM, et al. Pneumonitis in patients treated with Anti-Programmed Death-1/Programmed death ligand 1 therapy. $J$ Clin Oncol 2017;35:709-17.

11 Tang J, Pearce L, O'Donnell-Tormey J, et al. Trends in the global immuno-oncology landscape. Nat Rev Drug Discov 2018;17:783-4.

12 Brahmer JR, Lacchetti C, Schneider BJ, et al. Management of immune-related adverse events in patients treated with immune checkpoint inhibitor therapy: American Society of clinical oncology clinical practice guideline. J Clin Oncol 2018;36:1714-68.

13 Thompson JA, Schneider BJ, Brahmer J, et al. Management of Immunotherapy-Related toxicities, version 1.2019. J Nat/ Compr Canc Netw 2019;17:255-89.

14 Postow MA, Chesney J, Pavlick AC, et al. Nivolumab and ipilimumab versus ipilimumab in untreated melanoma. $N$ Engl $\mathrm{J}$ Med 2015;372:2006-17.

15 Naidoo J, Zhang J, Lipson EJ, et al. A multidisciplinary toxicity team for cancer Immunotherapy-Related adverse events. J Natl Compr Canc Netw 2019;17:712-20. 